\title{
Defining Control Strategies for MicroGrids Islanded Operation
}

\author{
J. A. Peças Lopes, Senior Member, IEEE, C. L. Moreira, and A. G. Madureira
}

\begin{abstract}
This paper describes and evaluates the feasibility of control strategies to be adopted for the operation of a microgrid when it becomes isolated. Normally, the microgrid operates in interconnected mode with the medium voltage network; however, scheduled or forced isolation can take place. In such conditions, the microgrid must have the ability to operate stably and autonomously. An evaluation of the need of storage devices and load shedding strategies is included in this paper.
\end{abstract}

Index Terms-Dynamic response, energy storage, frequency control, microgrid, power system dynamic stability, voltage control.

\section{INTRODUCTION}

$\mathbf{T}$ $\mathrm{HE}$ need of reducing $\mathrm{CO}_{2}$ emissions in the electricity generation field, recent technological developments in the microgeneration domain, and electricity business restructuring are the main factors responsible for the growing interest in the use of microgeneration [1], [2]. In fact, the connection of small generation units-the microsources (MS), with power ratings less than a few tens of kilowatts-to low voltage (LV) networks potentially increases reliability to final consumers and brings additional benefits for global system operation and planning, namely, regarding investment reduction for future grid reinforcement and expansion.

In this context, a MicroGrid (MG) can be defined as an LV network (e.g., a small urban area, a shopping center, or an industrial park) plus its loads and several small modular generation systems connected to it, providing both power and heat to local loads [combined heat and power (CHP)]. The Consortium for Electric Reliability Technology Solutions (CERTS) initiated the research on the impact of connecting large amounts of distributed energy resources (DER) to LV networks in order to enhance the reliability of the electric power system and developed the MG concept [1]. The MG is intended to operate in the following two different operating conditions.

- Normal Interconnected Mode - the MG is connected to a main MV network, either being supplied by it or injecting some amount of power into the main system.

Manuscript received September 12, 2005; revised November 3, 2005. This work was supported in part by the European Union (EU) within the framework of EU Project MicroGrids Contract ENK-CT-2002-00610 and in part by FCT-Fundação para a Ciência e a Tecnologia under Grant SFRH/BD/16473/2004. Paper no. TPWRS-00573-2005.

The authors are with the INESC Porto-Instituto de Engenharia de Sistemas e Computadores do Porto and FEUP-Faculdade de Engenharia da Universidade do Porto, Porto, Portugal (e-mail: jpl@fe.up.pt; cmoreira@ inescporto.pt; agm@inescporto.pt).

Digital Object Identifier 10.1109/TPWRS.2006.873018
- Emergency Mode - the MG operates autonomously, in a similar way to physical islands, when the disconnection from the upstream MV network occurs.

\section{A. General Overview}

It will not be common to find fully controllable synchronous generators in an MG, which are normally responsible for voltage and frequency control in conventional power systems. Most MS technologies that can be installed in an MG are not suitable for direct connection to the electrical network due to the characteristics of the energy produced. Therefore, power electronic interfaces $(\mathrm{dc} / \mathrm{ac}$ or ac/dc/ac) are required. Inverter control is thus the main concern in MG operation. In [3] and [4], a control scheme based on droop concepts to operate inverters feeding a standalone ac system is presented. This concept was further developed in this research (using two different inverter control schemes) to allow for MG islanded operation. The feasibility of this operational concept has been preliminarily described by the authors in [5].

Fault events that may lead to islanding of a distribution system are discussed in [6] assuming, however, that the MG (related in this case to an MV distribution system) comprises synchronous generators and grid power electronic interfaced generation units. In [7] and [8], the behavior of DER connected to distribution networks has also been addressed. However, the dynamics of the primary energy sources has not been considered, not allowing to obtain the full picture of the MG long-term dynamic behavior, which is largely influenced by the MS dynamics.

The feasibility of the MG islanding mode concept was laboratory tested in a prototype installed in the National Technical University of Athens (NTUA), which comprises a photovoltaic (PV) generator, battery energy storage, loads, and a controlled interconnection to an LV grid [9]. The converters used to couple the PV and the battery storage to the LV grid allow MG operation either in an LV grid interconnected mode or under islanded conditions. Also, experimental tests for islanding and synchronization were presented in [9].

The work described in this paper regards the evaluation, through numerical simulation, of the inverter-fed MG dynamic behavior under islanded operation for different load conditions and using two different control strategies. The impact of considering the primary energy source dynamics and the inverters contributions to faults are issues addressed in this paper. The robustness of the tested control strategies was evaluated for large disturbances taking place in the MV network, followed by a forced islanding of the MG. Since there is little inertia in the MG, and MS have small time constants, a combination of 


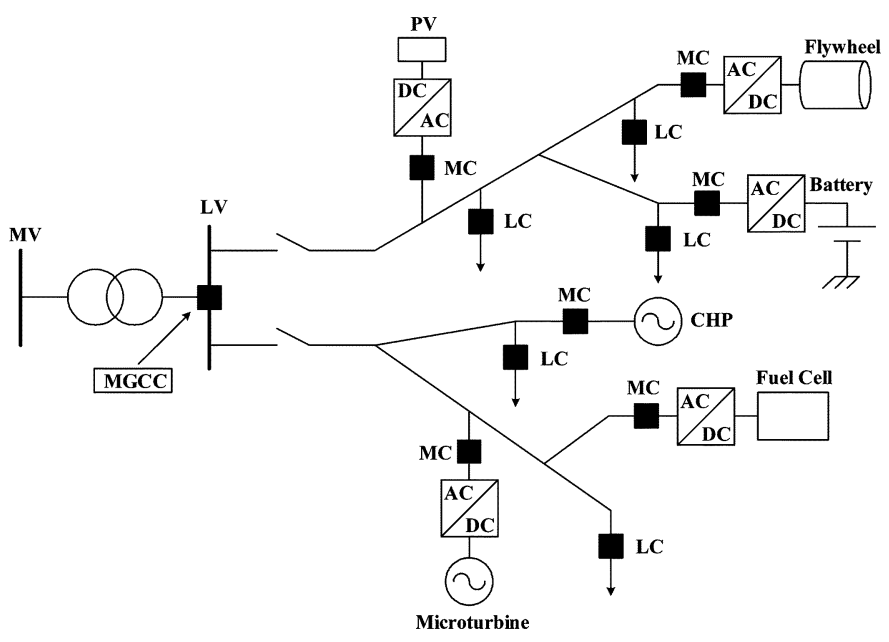

Fig. 1. MG architecture, comprising MS, loads, and control devices.

load shedding strategies and the use of storage devices were investigated in order to avoid large frequency and voltage excursions during the operating emergency mode conditions.

MatLab Simulink and its libraries (mainly the SimPowerSystems toolbox) were employed in order to develop a simulation platform suitable for identifying MG control requirements and evaluating MG dynamic behavior under several operating conditions. Different MS technologies were modeled and are considered to operate together in the simulation platform.

\section{MG ARCHITECTURE}

The MG concept adopted in this research involves an operational architecture, developed within the EU R\&D Microgrids project [2], [10], which is presented in Fig. 1. It comprises an LV network, loads (some of them interruptible), both controllable and noncontrollable MS, storage devices, and a hierarchical-type management and control scheme supported by a communication infrastructure used to monitor and control MS and loads.

The MG is centrally controlled and managed by a MicroGrid Central Controller (MGCC) installed at the MV/LV substation. The MGCC includes several key functions (such as economic managing functions and control functionalities) and heads the hierarchical control system. At a second hierarchical control level, controllers located at loads or at groups of loads [load controller (LC)] and controllers located at MS [microsource controller (MC)] exchange information with the MGCC that manages MG operation by providing set-points to both LC and MC. LC serve as interfaces to control loads through the application of an interruptibility concept that includes local load shedding schemes in emergency situations; MC control active and reactive power production levels at each MS.

The amount of data to be exchanged between network controllers is small, since it includes mainly messages containing set-points to $\mathrm{LC}$ and $\mathrm{MC}$, information requests sent by the MGCC to LC and MC about active and reactive powers, and voltage levels and messages to control MG switches. Also, the short geographical span of the MG may aid establishing a communication infrastructure using low-cost communications. The adoption of standard protocols and open technologies

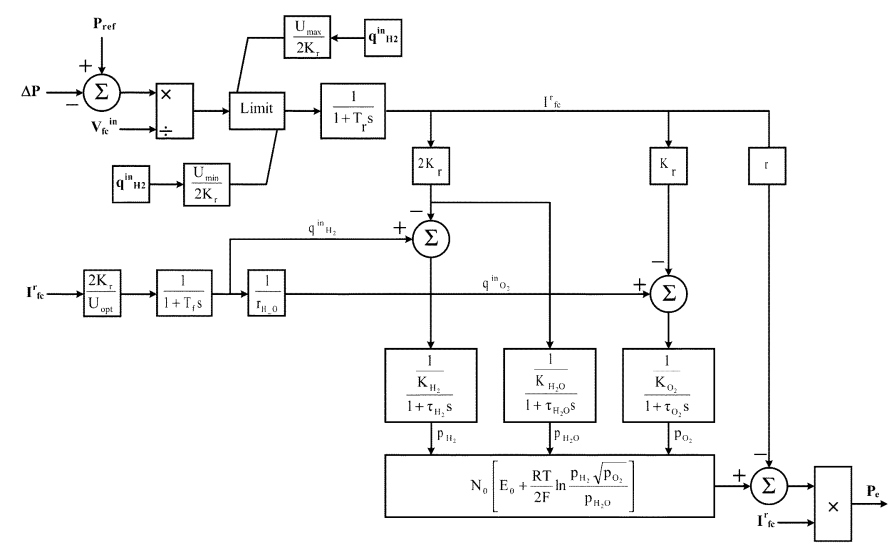

Fig. 2. SOFC block diagram model.

allows designing and developing modular solutions using off-the-shelf, low-cost, widely available, and fully supported hardware and software components. These solutions provide flexibility and scalability for future low-cost implementations.

\section{DynAmic Modeling OF COMPONENTS}

A set of models capable of simulating the response of the MG under several conditions was developed in order to analyze MG dynamic behavior.

\section{A. Microsource Modeling}

Several MS models, able to describe their dynamic behavior, have been developed from available literature, which include fuel-cells, microturbines, wind generators, and photovoltaic arrays.

For illustration purposes, some details on the dynamic model developed for the solid oxide fuel-cell (SOFC) are given next. The fuel-cell includes a fuel processor that converts the used fuel in hydrogen, a power section, where chemical reactions take place, and a power conditioner that converts de to ac power. The SOFC model adopted assumes several simplifications, such as fuel gases are considered to be ideal, it is sufficient to define only one single pressure value in the interior of the electrodes, the temperature in the fuel-cell is presumed to be always stable, only ohmic losses are considered, assuming that the working conditions are far away from the upper and lower extreme values of current, and the Nernst equation is assumed to be applicable. The complete model can be seen in the block diagram in Fig. 2. A complete description on the SOFC dynamic model and its parameters can be found in [11] and [12].

The GAST dynamic model [11] was adopted for the primary unit of microturbines, since these units are small simple-cycle gas turbines. Both high-speed single-shaft units (with a synchronous machine) and split-shaft units (using a power turbine rotating at $3000 \mathrm{rpm}$ and a conventional induction generator connected via a gearbox) were modeled. The single-shaft microturbine (SSMT) requires an ac/dc/ac converter for grid connection.

A wind generator was also included in the library of MS using for that purpose an induction generator directly connected to the network and represented by a fifth-order model, available in MatLab Simulink toolboxes. 
Concerning the PV generator, it was assumed that the array is always working at its maximum power level for a given temperature and irradiance. Basically, it is an empirical model based on experimental results as described in [13], where a detailed description on MS modeling adopted in the MG project can also be found.

\section{B. Storage Devices Modeling}

Due to the large time constants of the responses of some MS, such as fuel-cells and microturbines, storage devices must be able to provide the amount of power required to balance the system following disturbances and/or significant load changes.

Considering the time period of interest for analyzing MG dynamic behavior, storage devices, such as flywheels and batteries, are modeled as constant dc voltage sources using power electronic interfaces to be coupled with the electrical network (ac/dc/ac converters for flywheels and dc/ac inverters for batteries). These devices act as controllable ac voltage sources (with very fast output characteristics) to face sudden system changes such as in load-following situations. In spite of acting as voltage sources, these devices have physical limitations and thus a finite capacity for storing energy. The active power needed to balance generation and consumption inside the MG is injected into the LV grid using a proportional to frequency deviation control approach (with a specified droop characteristic).

\section{Inverter Modeling}

Two kinds of control strategies may be used to operate an inverter. The inverter model is derived according to the following control strategies.

- $P Q$ inverter control: the inverter is used to supply a given active and reactive power set-point.

- Voltage source inverter (VSI) control: the inverter is controlled to "feed" the load with pre-defined values for voltage and frequency. Depending on the load, the VSI real and reactive power output is defined.

In [14], more details on these control strategies can be found. It must be highlighted that, when analyzing the dynamic behavior of the MG, inverters are modeled based only on their control functions so that fast switching transients, harmonics, and inverter losses are neglected. This is the general procedure adopted, as described in [3], [6]-[8], [14], and [15].

1) $P Q$ Inverter Control: The PQ controlled inverter operates by injecting into the grid the power available at its input. The reactive power injected corresponds to a pre-specified value, defined locally (using a local control loop) or centrally from the MGCC.

The PQ inverter control is implemented as a current-controlled voltage source, as shown in Fig. 3. Current components in phase $\left(i_{\text {act }}\right)$ and quadrature $\left(i_{\text {react }}\right)$ with the inverter terminal voltage are computed based on a method presented in [9] for power calculation in single-phase inverters. Power variations in the MS induce a dc-link voltage error, which is corrected via the PI-1 regulator by adjusting the magnitude of the active current output delivered to the grid. The reactive power output is controlled via the PI- 2 regulator by adjusting the magnitude of the

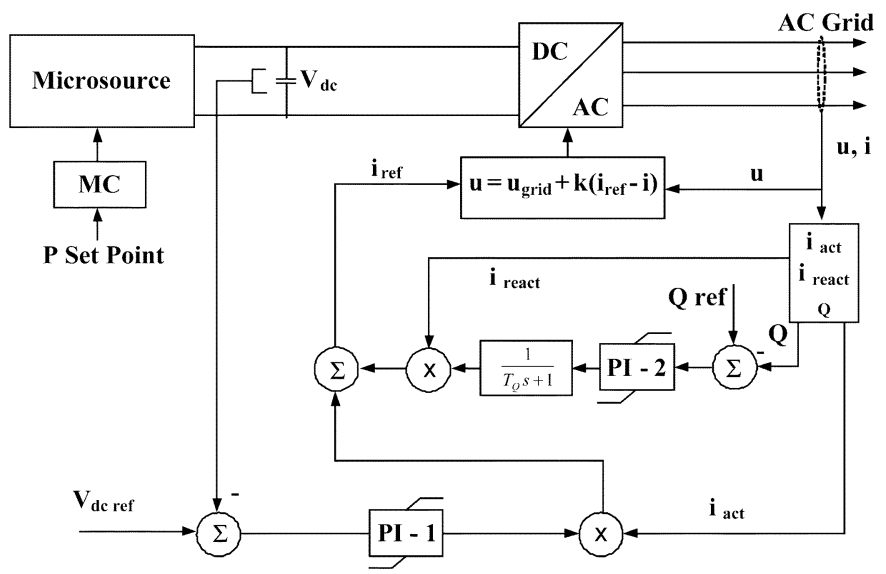

Fig. 3. PQ inverter control system.

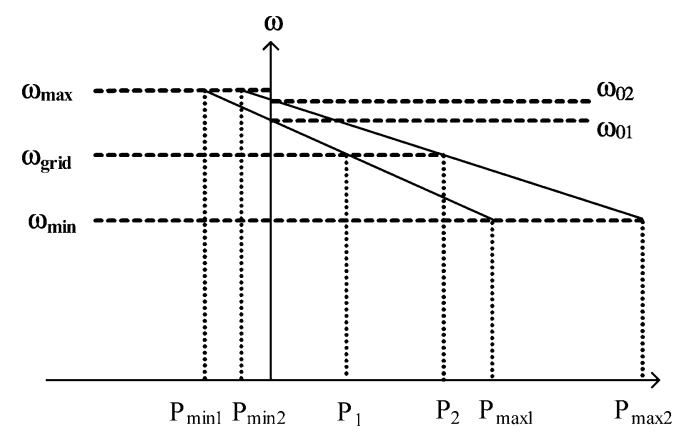

Fig. 4. Frequency versus active power droops.

inverter reactive current output. This inverter can be operated with a unit power factor or receive a set-point (locally or from the MGCC) for the output reactive power.

2) VSI Control: The VSI emulates the behavior of a synchronous machine, thus controlling voltage and frequency on the ac system [3], [4], [9]. The VSI acts as a voltage source, with the magnitude and frequency of the output voltage controlled through droops, as described in the following equations:

$$
\begin{aligned}
& w=w_{0}-k_{P} \times P \\
& V=V_{0}-k_{Q} \times Q
\end{aligned}
$$

where $P$ and $Q$ are the inverter active and reactive power outputs, $k_{P}$ and $k_{Q}$ are the droop slopes (positive quantities), and $\omega_{0}$ and $V_{0}$ are the idle values of the angular frequency and voltage (values of the inverter angular frequency and terminal voltage at no load conditions).

When a VSI is interconnected with a stiff ac system, characterized by an angular frequency $\omega_{\text {grid }}$ and terminal voltage $V_{\text {grid }}$, the voltage and frequency references are externally imposed [9]. In this case, the desired output powers $P_{1}$ and $Q_{1}$ can be obtained in the VSI output by adjusting the idle values of the angular frequency and voltage as follows (see Fig. 4):

$$
\begin{aligned}
& w_{01}=w_{\text {grid }}+k_{P} \times P_{1} \\
& V_{01}=V_{\text {grid }}+k_{Q} \times Q_{1} .
\end{aligned}
$$




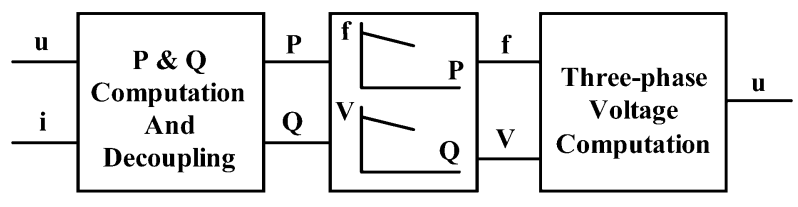

Fig. 5. VSI model.

If a cluster of VSI operates in a standalone ac system, frequency variation leads automatically to power sharing, such that for a system with $n$ VSI, the following equality stands:

$$
\Delta P=\sum_{i=1}^{n} \Delta P_{i}
$$

with $\Delta P_{i}$ being the power variation in the $i t h$ VSI. The frequency variation can be computed as

$$
\begin{aligned}
\Delta w & =w_{0 i}-k_{P i} \times P_{i}-\left[w_{0 i}-k_{P i} \times\left(P_{i}+\Delta P i\right)\right] \\
& =k_{P i} \times \Delta P i .
\end{aligned}
$$

Similar considerations can be made for the voltage/reactive power VSI control mode based on droops [3], [4]. However, as voltage has local characteristics, network cable impedances do not allow a precise sharing of reactive power among VSI.

A three-phase balanced model of a VSI implementing the described droop concepts was derived from a single-phase version presented in [16] and shown in Fig. 5. A complete description on VSI modeling can be found in [16] and [17]. The VSI terminal voltage and current are measured in order to compute active and reactive powers. This measuring stage introduces a delay for decoupling purposes. The output voltages are the reference signals that control the VSI switching sequence using a PWM modulation technique.

In terms of standalone ac system operation, this control principle allows the VSI to react to system disturbances (for example, load or generation changes) based on information available at its terminals [4]. In this way, the operation of a standalone ac system like an MG does not rely on fast communications between MS controllers, which could be impractical [3]. However, a communication infrastructure will be available in the MG, with the purpose of allowing an optimal management, not requiring fast communication capabilities.

3) Inverter Control During Transient Overloads and Short Circuits: Inverter overcurrents can be caused by transient overloads (due to the connection of a large amount of load in islanded mode) or by short circuits.

When moving to islanded operation or when a large load is connected during islanded operation, the initial high imbalance between local load and generation may lead to large frequency deviations and transient overload of the VSI, since this unit has a fast response to this type of situations. In order to overcome this problem, the following two solutions were adopted.

- Allow a temporary disconnection of less important loads (load shedding activated by under-frequency relays);

- Up-rate the VSI, allowing an overload situation during a certain time interval (at least greater than the one required for load shedding activation).
Due to the nature of the control of PQ inverters, they are not significantly affected by transient phenomena like load changes or power variations in renewable DER.

Conventional power plants comprising synchronous generators directly connected to the network provide large short-circuit currents, which are very helpful for fast and efficient fault detection and elimination. However, in an MG where generation units are mainly connected to the grid through power electronic interfaces, it is difficult to obtain high fault currents. Solid state switching devices used in inverters are selected based on voltage, current carrying capability (under certain cooling conditions for a defined switching frequency) and safe operating areas. The islanded system can ride through short-circuits if there is sufficient oversizing of power electronic interfaces, since they have no thermal short-term overcurrent capabilities, in contrast to synchronous generators. At the same time, a novel protection scheme for the MG must be developed using different types of relays and section breakers instead of conventional fuses. Accordingly, the following considerations are in order.

- The VSI was selected to be up-rated in order to provide a significant contribution to short-circuit currents (ranging from 3-5 p.u.) due to conventional overcurrent protection devices.

- PQ inverters can provide only a small amount of short circuit current (1.5-2 p.u.).

After a short-circuit, the time interval in which large current outputs are absorbed from the VSI is dependent on the impact caused by the dynamics of induction machines (operating as motors or generators). Induction machines can recover from short-circuits if the stability limit is not over-passed and enough reactive power is provided by external sources. In this case, the VSI will provide reactive power within the specified current limits and this behavior will be sustained until the induction machines recover. Thus, it is also important that the VSI are able to sustain overcurrents for a time interval larger than the one required for fault clearance.

The PQ inverter control scheme allows a simple control over the output inverter current during short-circuit conditions by limiting the total gain of the PI controllers shown in Fig. 2. Acting as a voltage source, the output current of a VSI tends to be very high (similarly to what happens in a conventional synchronous machine). In order to limit its output current, a control technique such as the one presented in Fig. 2 is used. The main difference is that in this case, the current reference has a maximum peak value dependent on the characteristics of the solid-state switches, and its frequency is imposed by inverter frequency/active power droop.

\section{Network and Load Modeling}

In this paper, the evaluation of the feasibility of MG islanded operation was performed through the analysis of the LV network dynamic behavior considering only three-phase balanced operation, despite the fact that this is not the most common situation in LV distribution networks. Two load types were considered: constant impedance loads (dependent on frequency and voltage) and motor loads (induction motor with nominal power ranging 
between 3 and $10 \mathrm{~kW}$ ). Electrical parameters of motor loads are typical values available in MatLab Simulink SimPowerSystems toolbox. As will be shown, load characteristics greatly influence the dynamic behavior of the MG, mainly under short-circuit conditions.

Controllable loads in the MG, available for load shedding purposes, have also been modeled. The amount of load to be shed is defined based on the amplitude of frequency deviation. In this study, motor loads have not been considered as sheddable loads.

\section{MG CONTROL FOR ISLANDED OPERATION}

Islanding of the MG can take place by unplanned events like faults in the MV network or by planned actions like maintenance requirements. In this case, the local generation profile of the $\mathrm{MG}$ can be modified in order to reduce the imbalance between local load and generation and reduce the disconnection transient [6]. In the presence of unplanned events like faults, $\mathrm{MG}$ separation from the MV network must occur as fast as possible. However, the switching transient will have great impact on MG dynamics.

If there are no synchronous machines to balance demand and supply, through its frequency control scheme, the inverters should also be responsible for frequency control during islanded operation. In addition, a voltage regulation strategy is required; otherwise, the MG might experience voltage and/or reactive power oscillations [18]. If a cluster of MS is operated within an MG and the main power supply (the MV network) is available, all the inverters can be operated in PQ mode, because there are voltage and frequency references. In this case, a sudden disconnection of the main power supply would lead to the loss of the MG, since there would be no possibility for load/generation balancing and therefore for frequency and voltage control. However, by using a VSI to provide a reference for voltage and frequency, it is thus possible to operate the MG in islanded mode, and a smooth moving to islanded operation can be performed without changing the control mode of any inverter [9]. As previously described, the VSI can react to network disturbances based only on information available at its terminals. This working principle of a VSI provides a primary voltage and frequency regulation in the islanded MG. After identifying the key solution for MG islanded operation, two main control strategies are possible: a) single master operation (SMO) or b) multi master operation (MMO). In both cases, a convenient secondary load-frequency control during islanded operation must be considered to be installed in controllable MS.

\section{A. Single Master Operation}

A general overview of an SMO is shown in Fig. 6. In this case, a VSI-acting as master-can be used as voltage reference when the main power supply is lost being that all the other inverters operated in PQ mode (slaves). Local MS controllers can receive information from the MGCC about the generation profile and control accordingly the corresponding MS.

\section{B. Multi Master Operation}

As described in Fig. 7, in a multi master approach, several inverters are operating as VSI with pre-defined frequency/active power and voltage/reactive power characteristics. The VSI can

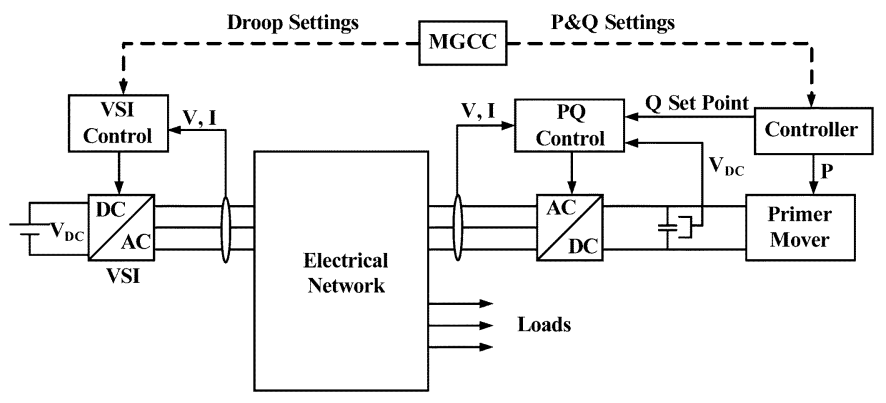

Fig. 6. Control scheme for SMO.

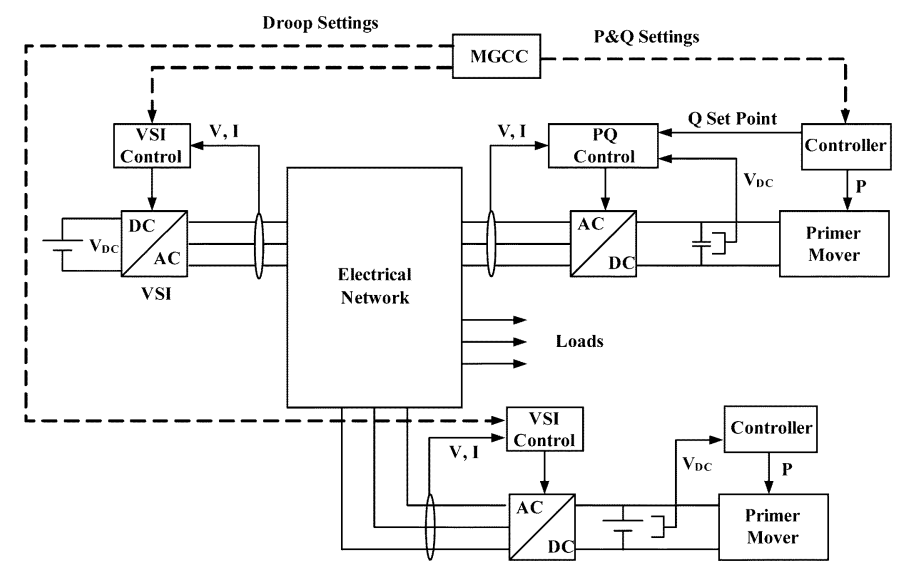

Fig. 7. Control scheme for MMO.

be coupled to storage devices (batteries or flywheels) or to MS with storage devices in the dc-link (batteries, super capacitors), which are continuously charged by the primary energy source. Eventually, other PQ-controlled inverters may also coexist. The MGCC can modify the generation profile by changing the idle frequency of VSI and/or by defining new set points for controllable MS connected to the grid through PQ-controlled inverters.

\section{Secondary Load-Frequency Control}

Equation (4) shows that the VSI active power output is proportional to the MG frequency deviation. If the MG frequency stabilizes in a value different from the nominal one (due to the use of only proportional droop controls), storage devices would keep on injecting or absorbing active power whenever the frequency deviation differs from zero. This should be only admissible during transient situations, where storage devices are responsible for the primary load-frequency control. Storage devices (batteries or flywheels with high capabilities for injecting power during small time intervals) have a finite storage capacity and can be loaded mainly by absorbing power from the LV grid. Therefore, correcting permanent frequency deviations during any islanded operating conditions should then be considered as one of the key objectives for any control strategy.

In order to promote adequate secondary control aiming to restore frequency to the nominal value after a disturbance, two main strategies can be followed: local secondary control, by using a PI controller at each controllable MS (Fig. 8) or centralized secondary control mastered by the MGCC. In both cases, target values for active power outputs of the primary energy sources are defined based on the frequency deviation error [19]. 


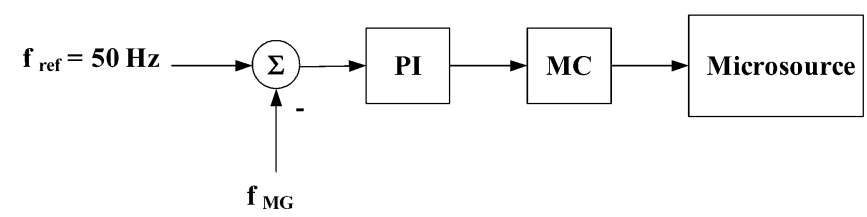

Fig. 8. Local secondary load-frequency control for controllable microsources.

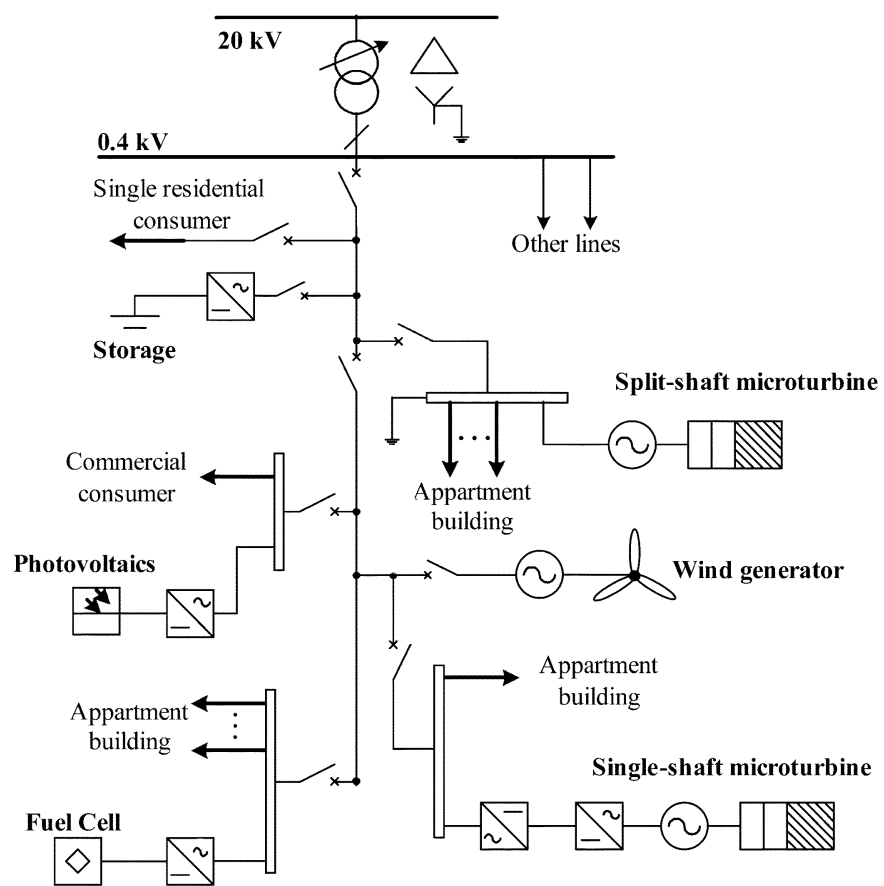

Fig. 9. LV test network.

For SMO, the target value is directly an active power set-point sent to the prime mover of a controllable MS (see Fig. 2), while for MMO, the target value can be both an active power set-point for a controllable MS connected to a PQ inverter or a new value for the idle frequency of a VSI.

\section{Simulation Platform}

A simulation platform under the MatLab Simulink environment was developed in order to evaluate the dynamic behavior of the MG. An LV test network was built based on a system defined by NTUA and used to test both presented control strategies (SMO and MMO). A detailed description of the test system and its electrical parameters can be found in [20]. Fig. 9 shows the MG single-line diagram. The implementation of the LV test network under the MatLab Simulink environment is shown in Fig. 10.

\section{RESULTS AND DISCUSSION}

Disconnection from the upstream MV network and load-following in islanded operation was simulated in order to understand the dynamic behavior of the MG and to evaluate the effectiveness of the developed control approaches. MG islanding can occur in two different situations (scheduled islanding and forced islanding) with possible faults taking place in the MV or LV grids. Due to space limitations, only results from forced islanding, due to faults in the MV grid, are described next. In

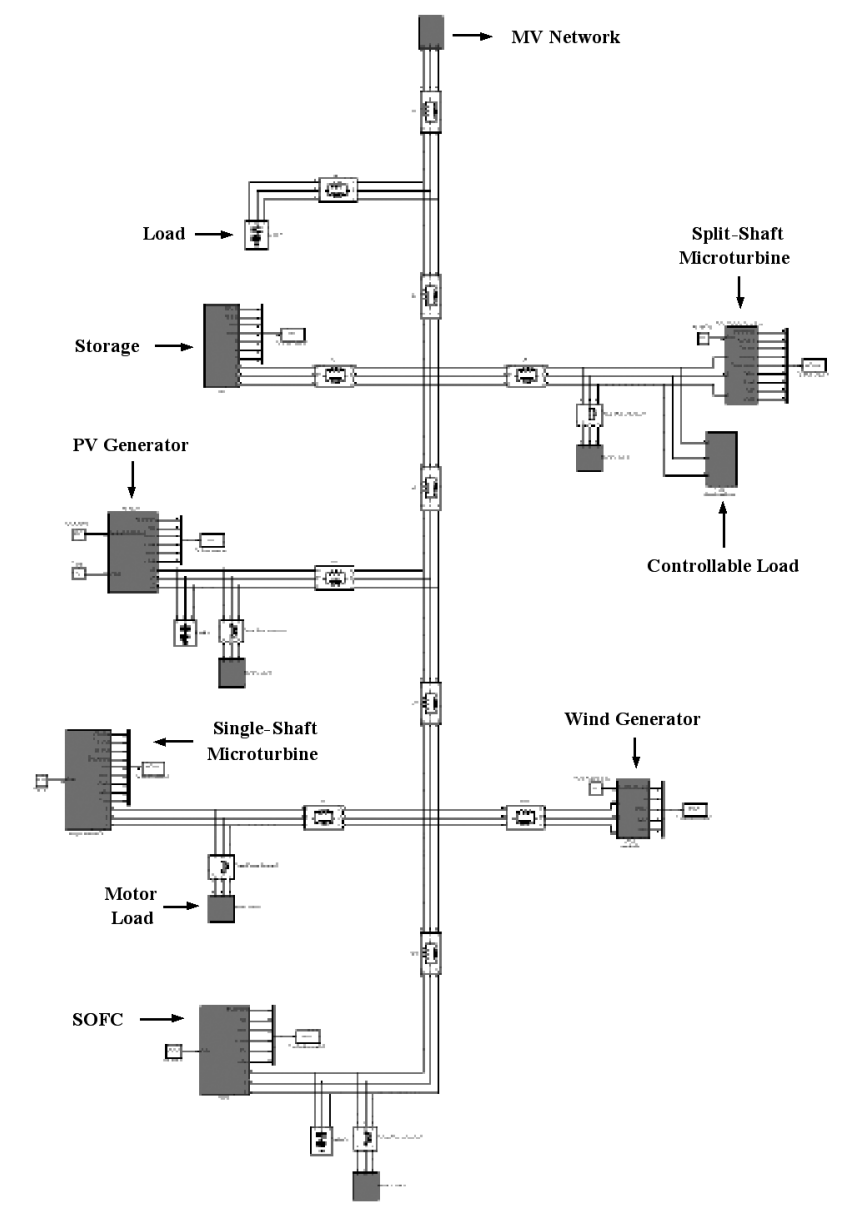

Fig. 10. LV network for the Matlab Simulink simulation platform.

this scenario, the SOFC and the SSMT are supposed to be the controllable MS used for the secondary load-frequency control using the scheme depicted in Fig. 8. Two load/generation scenarios using the single master operation strategy with a VSI and MMO were tested, with the corresponding results described next.

\section{A. Single Master Operation}

The scenario is characterized by a local load of $80 \mathrm{~kW}(65 \%$ of impedance type and $35 \%$ of induction motor type) and a local generation of $50 \mathrm{~kW}$ (high load scenario). A fault in the MV side occurred at $t=10 \mathrm{~s}$ followed by MG islanding $100 \mathrm{~ms}$ after.

Due to the large initial frequency deviation, a certain amount of load is automatically shed through the activation of under-frequency load shedding relays in order to aid frequency restoration. This load is later reconnected in small load steps, to avoid load disconnection by activating the under-frequency load shedding relays. Load reconnection in small steps allows the evaluation of the MG behavior under load-following conditions (see Fig. 11).

The principles described for current limitation in inverters can also be observed in Fig. 12 for the VSI for a PQ-controlled inverter (SOFC inverter).

The reacceleration of motor loads leads to a relatively slow ramping up of the voltage after fault clearing. Motor loads and asynchronous generators absorb high currents after disturbance 

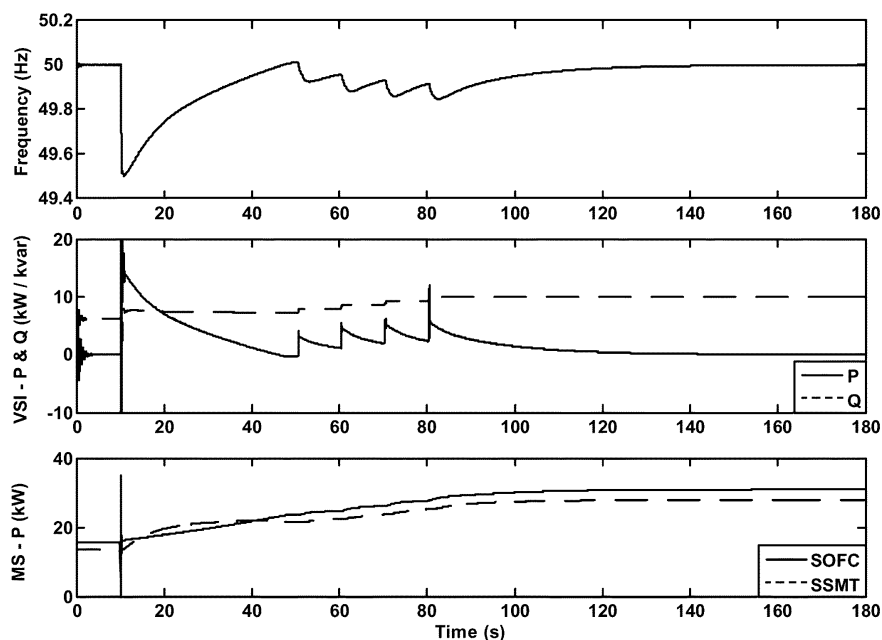

Fig. 11. MG frequency, VSI active and reactive power, and SOFC and SSMT active power (high load scenario).
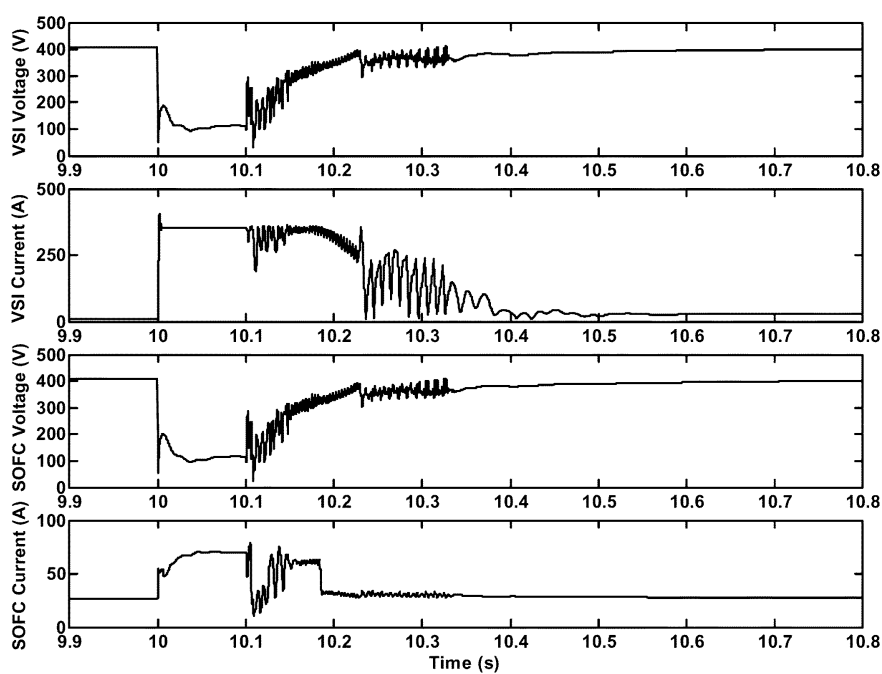

Fig. 12. Detail of the VSI and SOFC terminal voltages and currents during and subsequent to the MV fault and islanding procedure (high load scenario).

elimination, which lead to the activation of the short-circuit current limitation function in the VSI, as depicted in Fig. 12. After fault elimination, there is a transient period for restoring normal operation of asynchronous generators, which also contributes to the impact on inverter current and voltage, as it can be observed in Fig. 12 after $t=10.1 \mathrm{~s}$. Thus, a slower restoration of the MG voltage is observed. In this case, no motor loads were disconnected because all of them were able to successfully reaccelerate after fault elimination. Asynchronous generators (the split-shaft microturbine and the wind generator) were not disconnected in order not to lose generation.

In Fig. 13 are shown the time evolution of the terminal voltages in the VSI and SOFC and the generated reactive power in the SOFC and SSMT. The voltage/reactive power droop is the only mean used for voltage control during islanded operation. Thus, it is responsible for compensating reactive load transients as can be observed in Fig. 11. Reactive power in PQ-controlled inverters is kept constant (reactive power support) due to the control scheme described in Fig. 3.
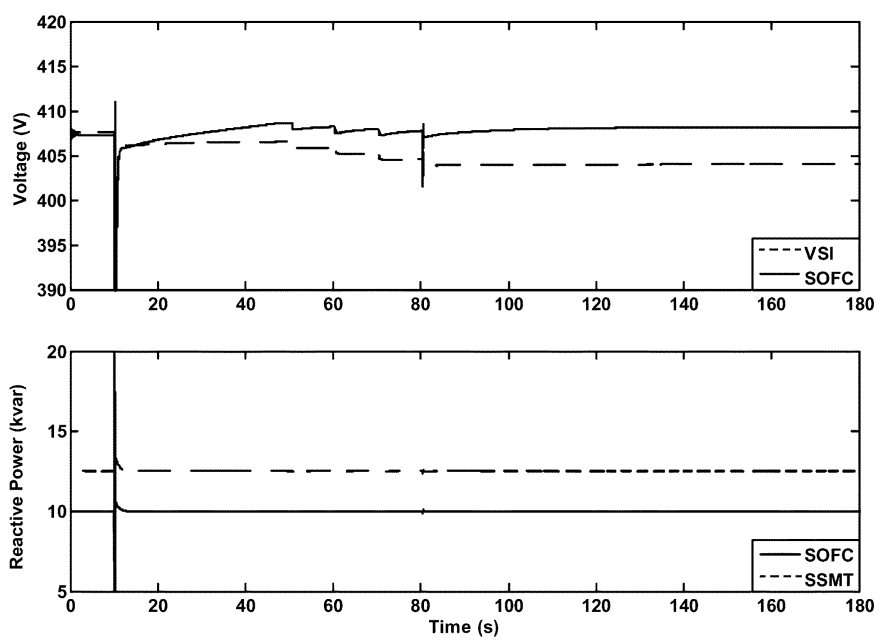

Fig. 13. Voltages and reactive powers (high load scenario).
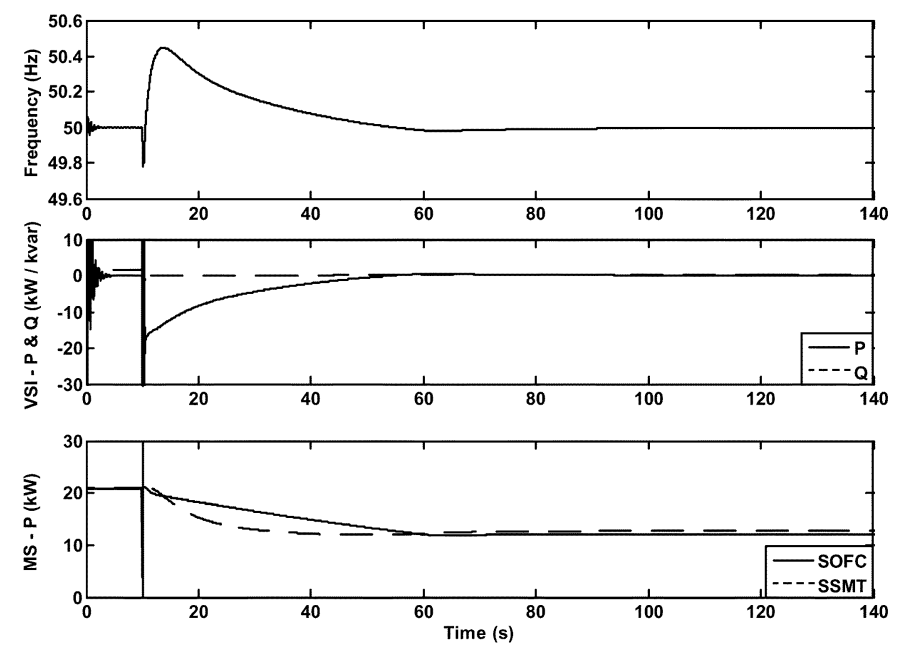

Fig. 14. MG frequency, VSI active and reactive power, and SOFC and SSMT active power (low load scenario).

A low load scenario with power being exported to the MV network was also tested. The scenario is characterized by a local load of $56 \mathrm{~kW}(70 \%$ of impedance type and $30 \%$ of induction motor type) and a local generation of $73 \mathrm{~kW}$. Fault conditions are those previously described.

The MG control strategies applied are valid for any operating conditions. The key issue for successful MG islanded operation is the management of load shedding and the state of charge of the storage devices. In this case, the MGCC should manage the state of charge of the storage so that it absorbs the power generation surplus after an unpredicted system islanding, as can be observed in Fig. 14. In both scenarios (high and low load conditions), a slow frequency restoration process that results from the large MS time constants can be observed. During this phenomenon, the VSI is responsible for a continuous matching between load and generation.

\section{B. Multi Master Operation}

In order to analyze the initial dynamic behavior of an MG under a multi master approach, the dynamics of the primary energy sources can be neglected due to the high storage capacity 

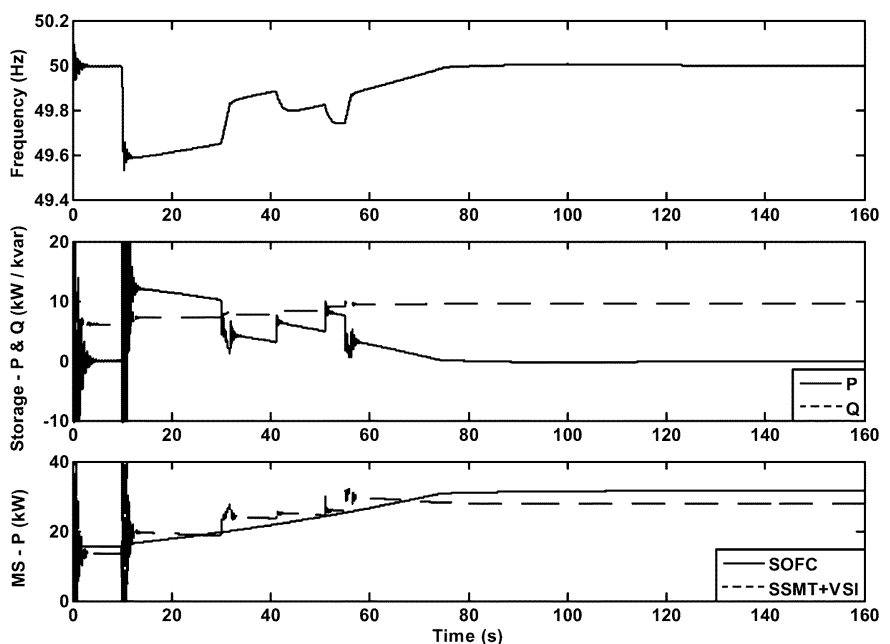

Fig. 15. MG frequency, VSI active and reactive power, and SOFC and SSMT active power (high load scenario).
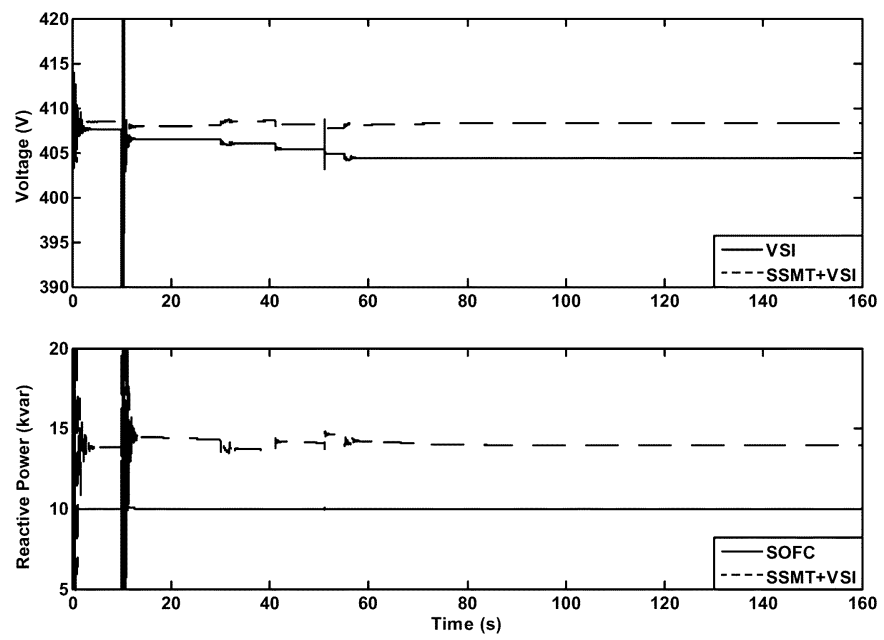

Fig. 16. Voltages and reactive powers.

assumed to be installed at their dc link [13]. Some microturbine manufactures install batteries on its dc link in order to provide black start capability and allow operation under islanded conditions [21]. In this case, it was assumed that the SSMT has a storage device installed on its dc link, and in this way, the inverter used to connect it to the LV grid can be operated as a VSI. In the high load scenario described for the single master, operation was used in this simulation.

Due to the existence of a higher storage capacity, system frequency is restored faster to its nominal value. The SSMT with storage devices in its dc link increases its output power in three steps: the first one corresponds to the contribution after MG islanding, which occurs due to the control scheme used in the VSI. The second and the third ones occur at $t=30 \mathrm{~s}$ and at $t=55 \mathrm{~s}$, respectively, as can be observed in Fig. 15. They were defined in advance based on the information about the load and generation imbalance with the MG before islanding takes place. The SOFC power output was controlled as depicted in Fig. 5 in order to restore MG frequency to the nominal value.

In this case, MG voltage support is assured by the voltage/reactive power droop controllers installed in the two VSI. As it can be observed in Figs. 15 and 16, there are no reactive power oscillations among MS, and MG stability is maintained. Proportional voltage control through droops is sufficient to maintain voltage levels within acceptable limits. However, reactive power sharing is not performed according to droops (similarly to what happens with active power) due to cable impedances.

\section{CONCLUSION}

This paper describes and evaluates the feasibility of the control strategies needed for MG islanded operation, where no directly grid-connected synchronous generators are used. From the analysis performed, the following main conclusions can be derived.

- Simulation results indicate that the forced islanding of the MG can be performed safely under several different power importing and exporting conditions.

- A fast elimination of a fault in the MV network is required; otherwise, motor loads may compromise the transition to islanded operation. Undervoltage load-shedding of large motor loads and induction generators could be an interesting possibility.

- Simulation results also indicate that both control strategies tested-SMO and MMO-are effective and ensure efficient and stable MG operation.

- The results obtained suggest that the management of storage devices are absolutely essential to implement successful control strategies for MG operation in islanded mode with the load shedding procedure assuming also very high importance to avoid fast and long frequency deviations.

\section{ACKNOWLEDGMENT}

The authors would like to thank all research teams of the MicroGrids project for valuable discussions.

\section{REFERENCES}

[1] R. Lasseter et al.. White Paper on Integration of Distributed Energy Resources-The CERTS MicroGrid Concept. [Online]. Available: http://certs.lbl.gov/pdf/LBNL_50 829.pdf.

[2] European Research Project MicroGrids. [Online]. Available: http://microgrids.power.ece.ntua.gr/.

[3] R. Lasseter and P. Piagi, "Providing premium power through distributed resources," in Proc. 33rd Hawaii Int. Conf. System Science, vol. 4, Jan. 2000.

[4] M. C. Chandorkar, D. M. Divan, and R. Adapa, "Control of parallel connected inverters in standalone AC supply systems," IEEE Trans. Ind. Appl., vol. 29, no. 1, pp. 136-143, Jan.-Feb. 1993.

[5] J. A. P. Lopes, C. L. Moreira, and A. G. Madureira, "Defining control strategies for analysing microgrids islanded operation," in Proc. IEEE St. Petersburg PowerTech, St. Petersburg, Russia, 2005.

[6] F. Katiraei, M. R. Iravani, and P. W. Lehn, "Micro-grid autonomous operation during and subsequent to islanding process," IEEE Trans. Power Del., vol. 20, no. 1, pp. 248-257, Jan. 2005.

[7] T. Tran-Quoc et al., "Dynamic analysis of an insulated distribution network," in Proc. IEEE Power Systems Conf. Expo., vol. 2, Oct. 10-13, 2004, pp. 815-821.

[8] R. Caldon, F. Rossetto, and R. Turri, "Analysis of dynamic performance of dispersed generation connected through inverters to distribution networks," in Proc. 17th Int. Conf. Electricity Distribution, Barcelona, Spain, May 12-15, 2003. 
[9] D. Georgakis, S. Papathanassiou, N. Hatziargyriou, A. Engler, and C. Hardt, "Operation of a prototype microgrid system based on micro-sources equipped with fast-acting power electronics interfaces," in Proc. IEEE 35th PESC, vol. 4, Aachen, Germany, 2004, pp. 2521-2526.

[10] J. A. P. Lopes, J. T. Saraiva, N. Hatziargyriou, and N. Jenkins. Management of microgrids. presented at JIEEC2003. [Online]. Available: http://microgrids.power.ece.ntua.gr/documents/Microgrids_management_jieec2003.pdf.

[11] Y. Zhu and K. Tomsovic, "Development of models for analysing the load-following performance of microturbines and fuel cells," Elect. Power Syst. Res., vol. 62, no. 1, pp. 1-11, May 2002.

[12] J. Padullés, G. W. Ault, and J. R. McDonald, "An integrated SOFC plant dynamic model for power systems simulation," J. Power Sources, vol. 86, no. 1-2, pp. 495-500, Mar. 2000.

[13] N. Hatziagyriou et al., "Modeling of micro-sources for security studies," in Proc. CIGRE Session, 2004.

[14] S. Barsali, M. Ceraolo, and P. Pelacchi, "Control techniques of dispersed generators to improve the continuity of electricity supply," in Proc. PES Winter Meeting, vol. 2, 2002, pp. 789-794.

[15] M. Y. El-Sharkh, A. Rahman, M. S. Alam, A. A. Sakla, P. C. Byrne, and T. Thomas, "Analysis of active and reactive power control of a standalone pem fuel cell power plant," IEEE Trans. Power Syst., vol. 19, no. 4, pp. 2022-2028, Nov. 2004.

[16] A. Engler, "Applicability of droops in low voltage grids," Int. J. Distrib. Energy Res., vol. 1, no. 1, Jan.-Mar. 2005.

[17] J. A. P. Lopes, C. L. Moreira, and F. O. Resende, "Microgrids black start and islanding operation," in Proc. 15th PSCC, Liège, Belgium, 2005.

[18] R. H. Lasseter and P. Piagi, "Microgrid: A conceptual solution," in Proc. 35th PESC, vol. 6, Aachen, Germany, Jun. 2004, pp. 4285-4290.

[19] A. Madureira, C. Moreira, and J. A. P. Lopes, "Secondary load-frequency control for microgrids in islanded operation," in Proc. Int. Conf. Renewable Energy Power Quality, Spain, 2005.

[20] S. Papathanassiou. Study-Case LV Network. [Online]. Available: http://microgrids.power.ece.ntua.gr/documents/Study-Case\%20LVNetwork.pdf.
[21] R. J. Yinger. Behavior of Capstone and Honeywell Microturbines During Load Changes. [Online]. Available: http://certs.lbl.gov/pdf/LBNL_49 095.pdf.

J. A. Peças Lopes (M'80-SM'94) received the electrical engineering degree and the Ph.D. degree, both in electrical engineering, from the University of Porto, Porto, Portugal, in 1981 and 1988, respectively. In 1996, he received the Aggregation degree from the University of Porto.

Currently, he is an Associate Professor with Aggregation in the Department of Electrical Engineering of the Faculty of Engineering, University of Porto. He is presently Coordinator of the Power Systems Unit of Instituto de Engenharia de Sistemas e Computadores do Porto (INESC Porto).

C. L. Moreira received the electrical engineering degree (five-year course) in 2003 from the Faculty of Engineering, Porto University, Porto, Portugal. He is currently working toward the Ph.D. degree in electrical engineering from Porto University and Instituto de Engenharia de Sistemas e Computadores do Porto (INESC Porto).

$\mathrm{He}$ is a Researcher in the Power System Unit of INESC Porto. His main research interests are focused on microgrids dynamics and control.

A. G. Madureira received the electrical engineering degree (five-year course) in 2003 from the Faculty of Engineering of Porto University (FEUP), Porto, Portugal. He is currently working toward the M.Sc. with Instituto de Engenharia de Sistemas e Computadores do Porto (INESC Porto) and FEUP.

$\mathrm{He}$ is a Junior Researcher in the Power System Unit of INESC Porto. 